the analyst and the geometer, the realm of irrational covariants.

Upon the question of courses of instruction I wish to formulate two propositions :

(1) A first or elementary course in invariant theory ought never to be restricted to binary forms.

(2) Preliminary to or concurrent with an advanced course, there should be given courses in the theory of substitution groups or abstract groups, and in the algebra of modular systems and of entire functions.

Evanston, ILL.,

August, 1898.

REYE'S GEOMETRIE DER LAGE.

Lectures on the Geometry of Position. By Theodor Reye, Professor of Mathematics in the University of Strassburg. Translated and edited by Thomas F. Holgate, M.A., Pн.D., Professor of Applied Mathematics in Northwestern University. Part I. New York, The Macmillan Company, 1898. 8vo, xix +248 pp.

The true geometry of position has hardly been accessible in English up to the present time. 'Townsend's Modern Geometry and Lachlan's Modern Pure Geometry are vitiated by the use of the circle, they are essentially metric; Cremona's Projective Geometry, in Leudesdorf's translation, is curiously uninteresting and unattractive, and does not seem to take the student sufficiently into the heart of the subject. Russell's Pure Geometry follows the French treatment of cross ratio, which is based on apparently metric relations, though it is shown that these relations are such that the metric quality is eliminated. Thus while it is a thoroughly useful book, it only gradually frees the student from the limitations of Euclidean geometry, instead of enabling him to walk at liberty from the first. It is possibly one of the easiest books to read on the subject; grafting the new ideas on to those already established, it expresses the unknown in terms of the known, whereas the more correct and satisfactory treatment, building up geometry $a b$ initio, is apt to strike a student at first as an elaborate and artificial expression of the known in terms of the unknown. But while the grafting of projective geometry on 
to metric may at first commend the subject, as the student advances and finds that the metric aspect is gradually disappearing, he cannot help feeling the essentially illogical nature of the method, namely, that the treatment is based on something that is afterwards shown to be irrelevant. It is a little too much like the game with which our elders were wont to puzzle our childhood, in which an elaborate series of arithmetical operations, performed on a secretly chosen number, brought out a result that had no relation to the initial number.

As regards the suitability of any particular presentation of a subject for teaching purposes, an appearance of strangeness in the preliminaries is a very small disadvantage, not to be compared with the feeling of uncertainty caused by a later discovery of a serious fault in the logic. Thus while the French treatment of modern geometry is natural and logical when the subject is approached analytically, it is to the standard German text books that we must turn at present for the pure geometrical treatment. Von Staudt and Reye are the names that at once suggest themselves; neither of these has been hitherto accessible in English, though Henrici's article on geometry in the Encyclopædia Britannica closely follows Reye's Geometrie der Lage (Leipzig, 1866-1892) ; I do not know of any English account of von Staudt's writings, the Geometrie der Lage (Nuremberg, 1847) and the Beiträge zur Geometrie der Lage (Nuremberg, 1856-1860). Probably Reye has thirty readers to von Staudt's one ; his book is to be obtained without trouble, while von Staudt's is hardly in circulation; it is clearly printed in well chosen type pleasant to the eye, and great attention has been paid to the spacing and paragraphing, in marked contrast to von Staudt; moreover, Reye's sympathetic style is such as to commend the subject. Hence it is not surprising that Professor Holgate, wishing to make some such presentation of modern pure geometry available for students that prefer to face their mathematical and linguistic difficulties separately, should take up Reye's Geometrie der Lage as a matter of course. This work has undoubtedly great merits as a text book; but the lecturer must be prepared to deal with some flagrant logical lapses. Intellectual sincerity forbids that these be passed by without notice; yet I have seen that a frank recognition of their existence shakes a student's faith in the author, and diminishes his interest in the subject. I was interested to find last year, in discussing the matter with a distinguished Italian geometer, that he has felt precisely this difficulty, so strongly that he 
has now adopted von Staudt in place of Reye with good results. As he remarked, von Staudt may be difficult, and may throw some hard work on the lecturer, but in arrangement and thought he is quite as interesting as Reye, and he is always absolutely logical.

It is with much diffidence and some feeling of ingratitude that I venture to criticise Reye's Geometrie der Lage, a book from which I have derived so much pleasure and profit. The objections urged relate only to the more elementary part, which can fairly be compared with von Staudt; without wishing in any way to disparage Reye, it does seem a cause for regret that von Staudt should be virtually ignored in college work; and the fact that there is now a readable English version of Reye will inevitably push von Staudt still more to one side.

Von Staudt seems to approach the subject in this manner. We have before us the visible universe; this, in so far as it is visible, we will treat geometrically. But we cannot handle any part of it, and so we cannot measure anything. The objects we see, or might see (for this is really what he means by denkbar at the beginning of the geometry) are bounded portions of space, hence solids ; from these we obtain bounding surfaces, then bounding lines, and finally bounding points; and each of these can move in the preceding one of the series, so generating it. Straight lines and planes are defined as lines and surfaces having a particular property. The objects of our researches are therefore solids, surfaces, lines, and points; and all visible relations of these are matters for investigation. Any one of these objects is considered as having an individual unalterable existence, and as having position, which can be changed. The fact that there are lines in a plane that do not intersect is derived from observation ; these lines are called parallel. Parallelism is shown to be equivalent to equality of direction, direction itself being accepted as a given idea, with no definition; it is shown that the direction of a line imposes on the line conditions that for all purposes are such as are imposed by a point. It is then shown that adjoining to the points already given by our view of the universe some others, namely, ideal points, the fact that lines and planes have a given direction and disposition (aspect) can be expressed by saying that they pass through certain of these ideal points. It is shown that the ideal points must be spoken of as all lying in a certain plane, the ideal plane or plane at infinity ; this gives us in every plane one ideal line, in every line one ideal point; 
and it is proved that for all purposes these ideal elements are to be treated in the same manner as those actually given us by the visible universe. Similarly imaginary, or feigned, elements are introduced and defined; and the proper form of reference to these feigned elements being investigated for every combination in which they can occur, it is found that they must be referred to in precisely the same manner as real elements. After thus justifying their treatment as actual geometrical elements, von Staudt explicitly extends the domain of the visible universe by adjoining to it these formal elements, both ideal and feigned. Thus his introduction of infinitely distant elements is absolutely logical; he adopts a particular phraseology to express a visible phenomenon, and proves that the verbal consequences of this phraseology lead us to correct conclusions. This is a perfectly logical method.

But in Reye's geometry, where the elements (point, straight line, and plane) are given in an arbitrary manner, without any statement that they visibly exist and without any definition, logic demands a different treatment. Nevertheless, in Lecture II, Reye speaks as though his geometry were derived from vision; he says that a case (of the intersection of two lines) is apparently exceptional because the point is lost to view, and then he introduces the line at infinity to account for this, and to show that the case is not really exceptional. This is altogether illogical. If the straight line at infinity is to be used at all, it ought to be postulated, absolutely and arbitrarily, in the same manner as the three elements. The same difficulty presents itself in connection with the idea of perpendicularity. The first use of this for purposes of argument* is on p. 115† (R. 108), where the nature of a right angle and the properties of a circle are assumed without question; we are not even told what definition of a circle is used, still less is any justification offered. The truth is, Professor Reye in his Geometrie der Lage has not the courage of his convictions. If his geometry is to be a purely logical cold-blooded system, with only intellectual justification or interest, built up from the three elements which alone he postulates. parallelism and perpendicularity have no place in it, and their surreptitious introduction discredits the whole system. To justify the recognition of these conceptions, something more must be

\footnotetext{
* The introduction of the circle on p. 49 (R. 49) is admissible, for it occurs in an avowedly metric supplement.

$\dagger$ The references to pages are here given in general for both the translation and the original, the latter being indicated as $R$.
} 
given, arbitrarily and absolutely ; and this something ought apparently to be a fixed quadric. This might perhaps be given in the first instance only for the plane, and in the degenerate form, as a pair of imaginary points, these determining the line at infinity in that plane ; but the treatment would be certainly more satisfactory and possibly more simple if the absolute were introduced at once in its most general form. Whether a purely logical and intellectual geometry, thus constructed, would ever appear to an average student as in any way applicable to anything, is altogether another question and quite irrelevant; the system would at any rate be precisely what it claimed to be-synthetic geometry, built up honestly from the given elements without any extraneous help.

Objection on the score of logic may be taken also to the handling of the principle of reciprocity. This principle is really involved in the stated properties of the undefined elements, point, line, and plane ; for it is mentioned in detail that the relation of point or line to point, line, and plane is precisely the same as that of plane or line to plane, line, and point, and thus if figures are built up from points, lines, and planes, other figures can be built up by the same law from planes, lines, and points. And yet on p. 30 (R. 29), Reye shrinks from assuming the general validity of the principle without demonstration, and says that it will be proved later; and on p. 102 (R. 97), in dealing with the polar relation in a plane, he says that this proves the principle of reciprocity for primitive forms of two dimensions.

But whatever objections may be urged against some things in the book, its general charm, to which is partly due its acceptation as the standard text book, is undeniable, and it was inevitable that sooner or later it should be brought out in English. Professor Holgate has translated Part I from the third edition (1886); doubtless Parts II and III, which carry the subject far beyond its elements, will follow. The division into lectures is retained, with the additional advantage over the German original of the numbering of the articles. Here and there it is difficult to determine on what principle this numbering has proceeded; $\$ \S 41$ and 42 on pp. 28,29 , should not have been separated ; $\$ 82$ on p. 55 is inserted in the middle of one of the lines of the original. The translation is distinctly readable, and is in general very carefully executed. Some Teutonisms are to be found, for example, bisection point (p. 107), and intersection point, involution curve, and involution cone (p. 152). Such constructions as "the infinitely distant 
point lies out in both directions upon the line' (p. 18), "we can associate in certain figures, to each vertex, $* * *$ a corresponding element of another"' (p. 34), "rays or planes * * * may be sectioned by a straight line" (p. 40), "relate the sheaves $* * *$ perspectively to the range of points" (p. 187), are hardly admissible. Weitlaüfig, as used on p. 37 (R. 36) is not exactly profuse, but rather diffuse or prolix ; on pp. $2,33,35$, and many others, wird should be will, not would, in accordance with English usage. These do not affect the meaning; but there are two passages where the precise sense of the original has been slurred in the translation. On p. 12 (R. 12,13) "we have included angles as part of a sheaf' is given as the equivalent of "wir $* * *$ den Winkel als Theil eines Büschels definirt haben ;" and on p. 130 (R. 121), where Reye evidently intends to define the class of a regulus, this being the first time he has occasion to make use of it, he says: "Die Regelfläche ist von der zweiten Classe," which is translated "The surface is thus of the second class," as if the class of a surface had already been defined.

These are comparatively slight defects, such slips as are almost sure to occur in a translation; they are perhaps small matters to single out for notice from 248 pages, but when a book is translated, the details of the translation invite criticism, however much we may appreciate the unselfishness of the translator in expending so much time and trouble on so altruistic a task. There remains however a more important matter to discuss, that of terminology. The whole question of nomenclature in mathematics is a difficult one; for as primarily disconnected subjects are found to overlap, the characteristic terms may turn out to be inconsistent. Thus it is impossible to say that no one individual shall be allowed to change an established term, for a change may be imperative. But certainly no one has a right to change a term without ample justification, and it is difficult to see what reasons Professor Holgate can adduce strong enough to justify him in discarding the well established and universally understood pencil (Büschel) in favor of sheaf, and replacing sheaf (Bündel) itself by bundle. Doubtless these terms are in themselves just as good ; but the others are already and have been for many years in possession ; they are perfectly distinctive and euphonious, hence the change is unnecessary, and therefore to be deprecated as likely to cause confusion. Another danger to be guarded against is the unnecessary naturalization of foreign words; sheet is as good as nappe (p. 93); skew is used for schief (p. 53), why not also for 
windschief (p. 26) instead of gauche? the two words have practically the same signification. The word Schein, so significant in the German, is apparently untranslatable ; Professor Holgate adopts projector as the equivalent; this answers sufficiently well in Reye, but misses the point in von Staudt's use of the word (Geometrie der Lage, §3, p. 12), where the reference is explicitly to the visual foundation of the geometry. The translator of von Staudt will be hard put to it to render the term adequately.

The book is clearly and accurately printed, but is spoilt for pleasant handling by its most unusual weight.

BRYN MAWR,

Charlotte Angas Scott.

\begin{abstract}
September, 1898.
\end{abstract}

\title{
BURKHARDT'S THEORY OF FUNCTIONS.
}

Funktionentheoretische Vorlesungen. Von HEINRICH BuRKHARDT. Erster Teil : Einführung in die Theorie der analytischen Functionen einer complexen Veränderlichen. Leipzig, Veit \& Co., 1897. 8vo, xii + 213 pp.

THE object of the author in writing the little volume before us has been to furnish an introduction to the theory of functions which is not confined to the presentation of the methods of any one school (Cauchy, Weierstrass, Riemann) but blends these methods as far as possible into an organic whole. The author has been very successful in making his book an introduction not merely to those parts of the theory which have long been classical (algebraic, elliptic, and Abelian functions) but also to the many other important developments of the last thirty years.* The mathematical public may well congratulate itself that a mathematician so thoroughly familiar with all sides of the subject as is Professor Burkhardt has undertaken the task of writing an elementary work along these lines.

We will briefly indicate the subjects treated.

Chapter $I$ is an excellent presentation of the elementary theory of complex numbers and their geometric representation, in which the author has wisely restricted himself to the ordinary complex numbers $a+b i$. It is interesting to

\footnotetext{
* We note, for instance, the introduction of the terms automorphic functions, fundamental region, and the proof and applications of the law of symmetry.
} 